\title{
Análise comparativa da estrutura de sistemas de indicadores de desenvolvimento sustentável: Programa Cidades Sustentáveis e ABNT NBR ISO 37120:2017 1
}

\section{Comparative analysis of the structure of sustainable development indicator systems: Sustainable Cities Program and ABNT NBR ISO 37120:2017}

\author{
Couto, E. A. 1; Di Gregorio, L. T.2;
}

1 Programa de Engenharia Urbana, Universidade Federal do Rio de Janeiro, Av. Athos da Silveira Ramos, 149, Cidade Universitária, Rio de Janeiro, Brasil, elisa_ac93@poli.ufrj.br

2 Programa de Engenharia Urbana, Universidade Federal do Rio de Janeiro, leandro.torres@poli.ufrj.br

\section{RESUMO}

O acelerado crescimento das cidades traz a cada dia novos desafios para o desenvolvimento urbano sustentável. Dessa forma, a gestão urbana e a boa governança se tornam fundamentais para a garantia da qualidade de vida nas cidades, tendo como importante ferramenta $o$ uso de indicadores de desempenho para o monitoramento e tomada de decisão. O presente trabalho tem como objetivo a comparação entre o Programa Cidades Sustentáveis e a ABNT NBR ISO 37120:2017, sistemas de indicadores de desenvolvimento sustentável. O estudo é parte de uma pesquisa de mestrado em curso e utiliza dados de um trabalho de conclusão de curso para avançar na discussão do tema. Para a análise comparativa entre os sistemas, foram avaliados os perfis dos indicadores, reagrupando-os nos mesmos eixos temáticos, a partir de sua natureza. Foram, ainda, confrontados os resultados da aplicação das metodologias para o Rio de Janeiro. Como resultado observa-se que, apesar de determinadas diferenças de abordagem, as metodologias apontam para semelhanças quanto ao diagnóstico da transparência das informações para a capital fluminense. Conclui-se que a escolha por um conjunto de indicadores dependerá do alcance, detalhamento e credibilidade almejados e que os indicadores são importantes instrumentos para a gestão urbana e promoção da governança.

Palavras-chave: Indicadores de desenvolvimento sustentável, Gestão urbana, Governança, Cidades sustentáveis, ISO 37120

\section{ABSTRACT}

The accelerated growth of cities engenders new issues to sustainable urban development. In this way, urban planning and good governance become fundamental for ensuring the quality of life in cities, in order that the use of indicators can play an important role in monitoring and

${ }^{1}$ COUTO, E. A.; DI GREgORIO, L. T. Análise comparativa da estrutura de sistemas de indicadores de desenvolvimento sustentável: Programa Cidades Sustentáveis e ABNT NBR ISO 37120:2017. In: II SIMPÓSIO NACIONAL DE GESTÃo E ENGENHARIA URBANA: SINGEURB, 2019, São Paulo. Anais... Porto Alegre: ANTAC, 2019. 
decision making. This work aims to compare the Sustainable Cities Program and ABNT NBR ISO 37120:2017, sustainable development indicator systems. The study is part of an ongoing master's degree research and uses data from an undergraduate project to move forward in the topic discussion. For the comparative analysis between the different systems, the indicators profiles were evaluated, regrouping them in the same thematic axes, based on their nature. The results of the methodologies implementation for Rio de Janeiro were also confronted. As a result, despite certain differences in approach, the methodologies pointed to similarities in the diagnosis of information transparency for the city of Rio de Janeiro. It is concluded that the choice of a set of indicators will depend on the scope, detail, and credibility desired and that the indicators are important tools for urban management and governance promotion.

Keywords: Sustainable development indicators, Urban management, Governance, Sustainable cities, ISO 37120

\section{INTRODUÇÃO}

Com o crescimento acelerado das cidades ocasionado pelo constante aumento da população urbana, a qual estima-se que ultrapasse $90 \%$ da população brasileira em 2030 (ONU, 2016), surgem novos desafios acerca da sustentabilidade desse desenvolvimento. A sustentabilidade, nesse contexto, consiste na harmonia entre os diversos fatores promotores do desenvolvimento, de forma a atender as necessidades presentes e assegurar 0 atendimento das futuras (CMMAD, 1988).

Visto que o desenvolvimento sustentável é um processo a longo prazo, no qual a sociedade deve ser instruída através de políticas públicas (BEZERRA; BURSZTYN, 2000) e que governança é a "capacidade de decidir e implementar políticas públicas que atendam às necessidades da população" (PALUDO, 2012), a governança é essencial para uma gestão urbana sustentável.

A governança urbana participativa surge em um contexto de retração do Estado devido ao seu baixo desempenho na resolução dos problemas urbanos, incorporando a sociedade civil e cidadãos nessa dinâmica (FREY, 2007). Além da participação da sociedade, as diretrizes da governança pública passam pela transparência e monitoramento do desempenho das políticas praticadas (BRASIL, 2017). A Figura 1 apresenta a base para os mecanismos de governança, que devem estar alinhados aos princípios e diretrizes da boa governança.

Figura 1 - Mecanismos, instâncias e práticas de governança

\section{Requisitos mínimos dos mecanismos, instâncias e práticas de governança}

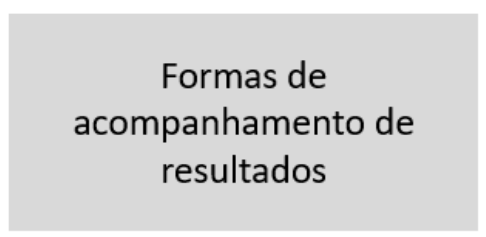

$$
\begin{gathered}
\text { Soluções para melhoria do } \\
\text { desempenho das } \\
\text { organizações }
\end{gathered}
$$

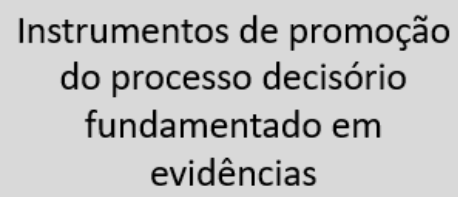

Fonte: BRASIL (2017). Adaptado pelos autores.

Nesse sentido, arcabouços de indicadores de sustentabilidade funcionam como ferramentas fundamentais para a governança e monitoramento de desempenho (FURTADO, 2009). Os indicadores também auxiliam nas tomadas de decisão, visto que estes simplificam a comunicação de fenômenos complexos, apontando para tendências e progressos ao longo do tempo (GOMES; MARCELINO; ESPADA, 2000), além de contribuírem, por meio de sua divulgação, para a transparência das informações.

O presente trabalho tem por objetivo a comparação entre a estrutura do conjunto de indicadores de desenvolvimento sustentável do Programa Cidades Sustentáveis e da ABNT NBR ISO 37120:2017, a fim de analisar a abordagem desses sistemas e a capacidade destes de avaliar a transparência dos municípios. Parte-se dos resultados da aplicação dos diferentes sistemas de indicadores para a cidade do Rio de Janeiro, que ocupa a $23^{a}$ posição no ranking 
de transparência entre as capitais, segundo a avaliação da Escala Brasil Transparente (EBT) (BRASIL, 2018).

\section{INDICADORES PARA CIDADES SUSTENTÁVEIS}

A criação de indicadores capazes de monitorar o desenvolvimento sustentável foi defendida pela Agenda 21 (CNUMAD, 1995), dando origem ao surgimento de diversos sistemas de indicadores no Brasil e no mundo.

Dentre as metodologias de indicadores voltadas para a avaliação da sustentabilidade nas cidades, no contexto brasileiro, destacam-se o Programa Cidades Sustentáveis e a norma ABNT NBR ISO 37120:2017 "Desenvolvimento sustentável em comunidades - indicadores para serviços urbanos e qualidade de vida".

O programa Cidades Sustentáveis foi lançado em parceria pela Rede Nossa São Paulo, a Rede Social Brasileira por Cidades Justas e Sustentáveis e o Instituto Ethos, em 2011, e dispõe de uma plataforma que abriga os dados de diversas cidades (PROGRAMA CIDADES SUSTENTÁVEIS). Já a NBR ISO 37120:2017 é resultado da tradução e adaptação da norma internacional ISO 37120:2014 pela Associação Brasileira de Normas Técnicas (ABNT, 2017). A norma ISO passou por uma atualização em 2018, ainda sem versão brasileira.

No caso da aplicação da ISO 37120, a cidade só é inserida na plataforma online quando certificada pelo World Council on City Data (WCCD), o que exige uma auditoria externa para verificação dos dados fornecidos.

As características dos sistemas de indicadores analisados no presente estudo estão resumidas no Quadro 1.

Quadro 1 - Caracterização dos sistemas de indicadores

\begin{tabular}{|c|c|c|}
\hline & Programa Cidades Sustentáveis & ABNT NBR ISO 37120:2017 \\
\hline Ano de criação & 2011 & 2017 (no Brasil) \\
\hline Aplicação & Cidades brasileiras & $\begin{array}{l}\text { Cidades, municipalidades e } \\
\text { governos locais ao redor do } \\
\text { mundo }\end{array}$ \\
\hline Número de indicadores & 260 & 100 \\
\hline Eixos temáticos & 12 & 17 \\
\hline Periodicidade & Anual & Anual \\
\hline Tipo de indicadores & Quantitativos (81\%) e qualitativos (19\%) & Quantitativos (100\%) \\
\hline Informações & $\begin{array}{c}\text { Fórmulas; relação com o ODS e meta } \\
\text { da agenda 2030; exemplos mundiais } \\
\text { de boas práticas (por eixo) }\end{array}$ & $\begin{array}{l}\text { Fórmulas; definições } \\
\text { detalhadas; fonte de dados; } \\
\text { notas adaptadas para o Brasil }\end{array}$ \\
\hline Plataforma & Plataforma Cidades Sustentáveis & WCCD \\
\hline
\end{tabular}

Fonte: Os autores

\section{METODOLOGIA}

Para a elaboração do presente trabalho, foi realizada uma pesquisa exploratória a respeito dos conjuntos de indicadores de desenvolvimento sustentável estabelecidos pelo Programa Cidades Sustentáveis e pela norma ABNT NBR ISO 37120:2017. 
Para o Programa Cidades Sustentáveis, a metodologia já havia sido aplicada pela prefeitura do Rio de Janeiro em 2017 e os dados disponibilizados na plataforma do Programa, de onde foram extraídas as informações. Já para a obtenção dos indicadores para a capital fluminense segundo a NBR ISO 37120, foi aplicada a metodologia da norma e foram calculados os indicadores a partir dos dados disponíveis para a população (COUTO, 2018).

Para o cálculo destes dados foi realizada uma pesquisa documental, por meio de censos, estudos e inventários de mais de 15 órgãos e organizações. Devido a periodicidade do recenseamento no Brasil, o ano de referência para os indicadores do Rio de Janeiro é 2010.

De forma a possibilitar a comparação entre os diferentes sistemas de indicadores, eles foram organizados nos mesmo eixos temáticos. Optou-se por seguir a organização da NBR ISO 37120 , assim os 260 indicadores do Programa Cidades Sustentáveis foram reagrupados nas 17 seções da norma, de acordo com a natureza dos indicadores. Em casos em que a classificação não era evidente, foram feitas algumas considerações. Dessa forma, indicadores relacionados a equipamentos culturais foram considerados no eixo da educação e dados de violência foram agrupados como segurança.

A partir desse rearranjo, foi comparada a distribuição dos indicadores por tema, assim como a quantidade de indicadores disponíveis para a cidade do Rio de Janeiro dentro de cada eixo, considerando que todos possuem o mesmo peso.

\section{RESULTADOS}

\subsection{Distribuição dos indicadores por eixos temáticos}

A distribuição percentual dos indicadores em cada eixo temático para os conjuntos de indicadores da norma e do Programa Cidades Sustentáveis, segundo as seções da NBR ISO 37120, está apresentada na Figura 2.

Figura 2 - Percentual de indicadores por eixo temático

\section{Indicadores por Eixo Temático}

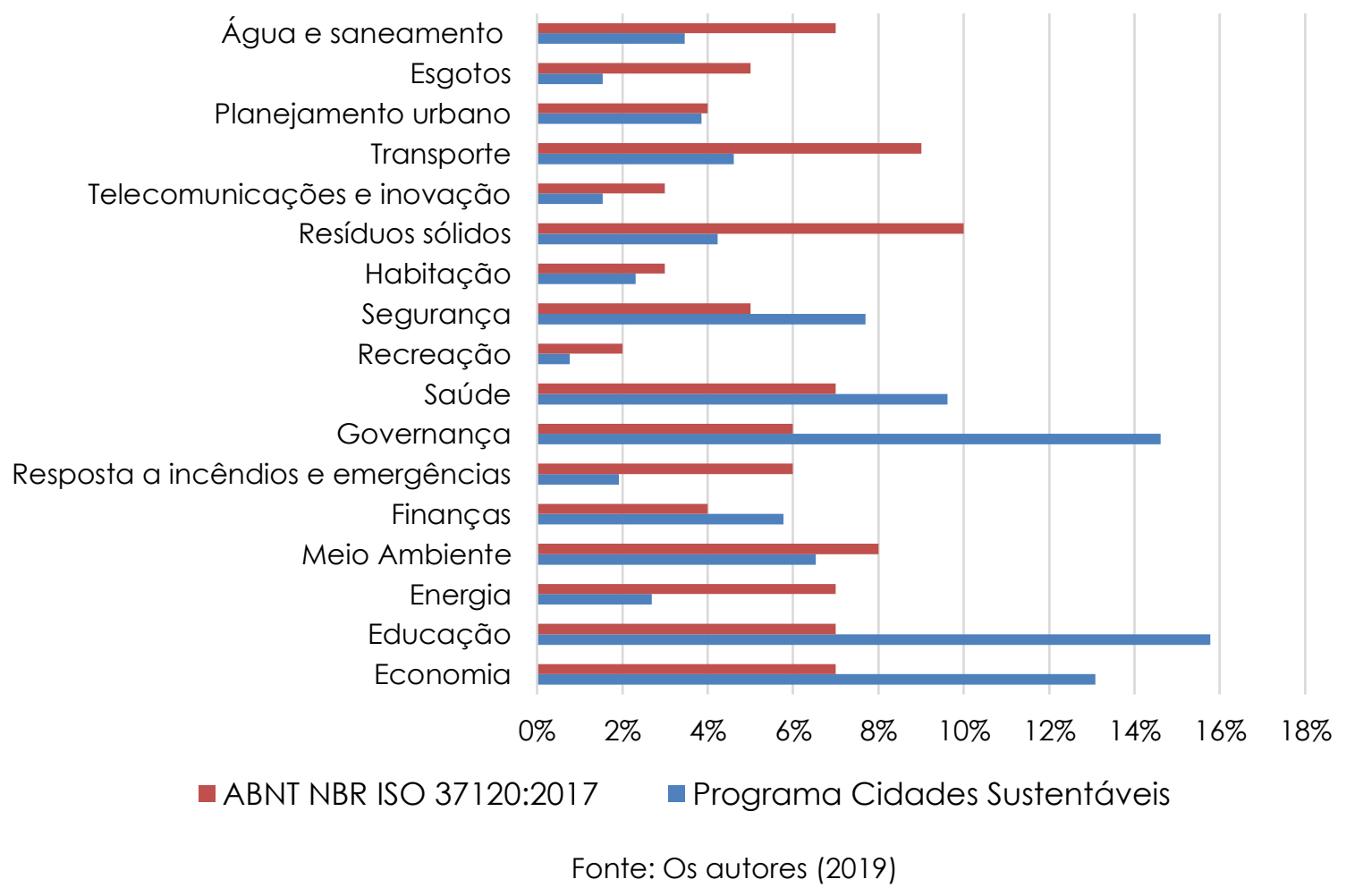


Observa-se que a NBR 37120 tem seus indicadores de forma mais distribuída, enquanto o Programa Cidades Sustentáveis foca em determinados eixos, de forma que os temas voltados para educação, governança, economia e saúde somam mais de $50 \%$ do total de indicadores. À exceção da saúde, estes são eixos que apresentam grandes discrepâncias entre os sistemas comparados, com diferenças entre 6 e 9 pontos percentuais.

Na educação, a norma aborda a população matriculada em escolas e a taxa de sobrevivência, enquanto o sistema brasileiro detalha questões como a demanda de vagas, formação dos professores e desempenho dos alunos segundo avaliações nacionais.

Em relação à governança, a norma traz indicadores sobre a participação dos eleitores, a representação das mulheres e casos de corrupção. Por sua vez, além da questão de gênero e corrupção, o Programa Cidades Sustentáveis discute transparência, acesso à justiça e a existência de ações e campanhas nesse sentido. Dos 38 indicadores existentes, 20 são qualitativos.

Na economia, ambos os sistemas tratam do desemprego e da pobreza. Enquanto a NBR 37120 foca nas empresas, o Programa Cidades Sustentáveis destrincha o PIB em algumas atividades, além de apresentar indicadores sobre desigualdade de renda e gênero e trabalho escravo.

O tema de resíduos sólidos é o mais representativo para a NBR 37120, correspondendo a 10\% de todos seus indicadores, contra $4 \%$ no sistema brasileiro. A principal diferença é que no primeiro é detalhada a forma de disposição final dos resíduos, enquanto no segundo o foco são as ações para sua reciclagem e recuperação.

A seção de planejamento urbano apresentou aproximadamente a mesma proporção, cerca de $4 \%$ dos indicadores, em ambos os sistemas. Em outros eixos, como meio ambiente, recreação, habitação e telecomunicações e inovação, a diferença é de apenas 1\%. Apesar da semelhança na proporção de indicadores, as abordagens em relação ao meio ambiente são distintas, uma vez que a metodologia brasileira trata das áreas de preservação e legislação ambiental, deixando de discutir aspectos da polvição sonora e do ar, contidos na norma.

\subsection{Disponibilidade de indicadores para o município do Rio de Janeiro}

Em relação aos indicadores disponíveis para o Rio de Janeiro, o Programa Cidades Sustentáveis dispõe de cerca de $63 \%$ do total, ao passo que a aplicação da NBR 37120 resultou na obtenção de $60 \%$ dos indicadores da norma. A proporção dos indicadores disponíveis em relação ao total por eixo, para cada metodologia, está indicada na Figura 3.

Nota-se que nas seções de esgotos, água e saneamento e segurança, a disponibilidade de indicadores para o Rio de Janeiro segundo a NBR 37120 é superior entre 40 e 50 pontos percentuais. Já o Programa Cidades Sustentáveis disponibilizou mais indicadores que a norma na seção de energia, $86 \%$ e $43 \%$, respectivamente. Para a recreação, a discrepância percentual é grande, mas os indicadores são pouco representativos.

Apesar das duas metodologias apresentarem alguns indicadores similares nos eixos de esgotos e água e saneamento, houve divergências quanto a sua disponibilidade. É o caso da população atendida por rede de esgoto, porcentagem de esgoto sem tratamento, perdas de água e consumo de água per capita, informações fornecidas pela agência responsável pelo serviço e indisponíveis entre os dados do sistema brasileiro.

Na seção de segurança, o Programa Cidades Sustentáveis trata o tema de forma mais ampla, trazendo indicadores sobre crimes sexuais, violência contra a mulher, idosos, crianças e adolescentes. A baixa disponibilidade aponta para a dificuldade de obtenção dessas informações. Já quanto aos indicadores da norma, o entrave foi na aquisição de dados da polícia, como efetivo e tempo de resposta.

Por outro lado, a proporção de indicadores disponíveis apresentou mais similaridades para os eixos de telecomunicações e inovação, resíduos sólidos e governança, em que a diferença 
entre os conjuntos de indicadores não ultrapassa $5 \%$.

Figura 3 - Percentual de indicadores disponíveis para o Rio de Janeiro

\section{Proporção de Indicadores Disponíveis para o Rio e Janeiro}

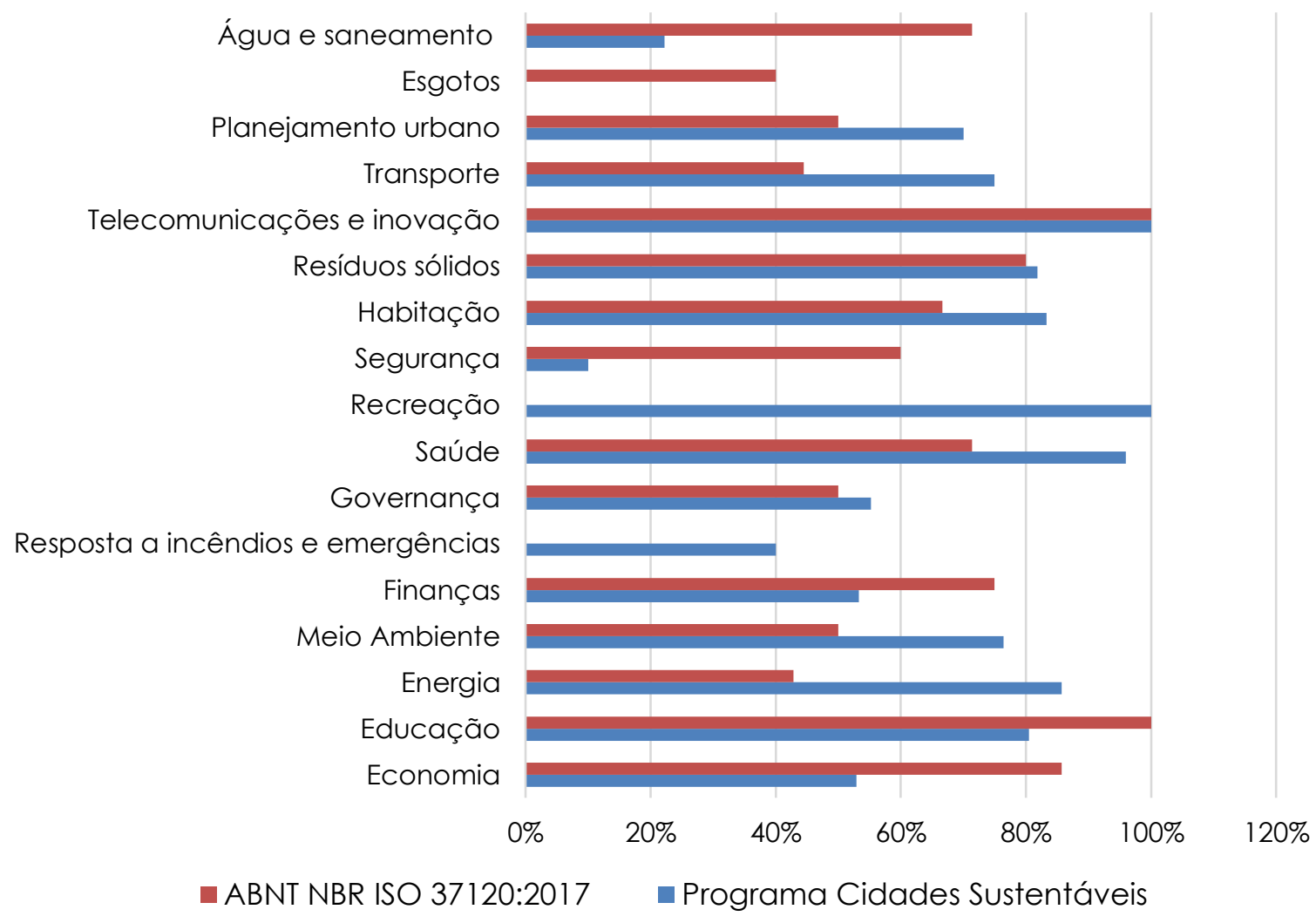

Fonte: Os autores (2019)

\section{CONCLUSÕES}

Através da comparação entres os sistemas avaliados, pode-se concluir que a NBR ISO 37120 apresenta indicadores mais globais, enquanto o Programa Cidades Sustentáveis aborda os temas de forma mais detalhada, limitando seu alcance. Apesar da norma possuir indicadores mais generalistas, a obrigatoriedade de auditoria para a certificação das cidades garante rigor na aplicação da metodologia e maior confiabilidade das informações.

Embora tenham sido apontadas diferenças na abordagem e distribuição dos indicadores ao longo dos eixos temáticos, a pesquisa apontou similaridades entre os dois conjuntos de indicadores analisados, principalmente no que diz respeito à disponibilidade de dados para o Rio de Janeiro. O resultado corrobora a avaliação da EBT para a cidade, indicando para a pertinência dos sistemas no diagnóstico da transparência do município, princípio da governança.

Além de se tratarem de um instrumento de acompanhamento do desempenho da cidade quanto ao seu desenvolvimento sustentável de forma geral, esses indicadores auxiliam no diagnóstico das práticas de governança, em seus respectivos eixos. Processos participativos de governança podem, ainda, potencializar o alcance dos indicadores ao complementar a interpretação destes com as visões dos múltiplos atores da sociedade. 


\section{REFERÊNCIAS}

ABNT ASSOCIAÇÃO BRASILEIRA DE NORMAS TÉCNICAS. NBR ISO 37120: Desenvolvimento sustentável de comunidades - Indicadores para serviços urbanos e qualidade de vida. Rio de Janeiro, 2017.

BEZERRA, M. C. L.; BURSZTYN, M. Ciência e tecnologia para o desenvolvimento sustentável. Brasília: Ministério do Meio Ambiente e dos Recursos Naturais Renováveis: Consórcio CDS/ UNB/ Abipti, 2000.

BRASIL. Controladoria-Geral da União. Portal da Transparência. Escala Brasil Transparente. Brasília, 2018. Disponível em:

$<\mathrm{http}$ //transparencia.gov.br/brasiltransparente? ordenarPor=posicao\&direcao=asc $>$. Acesso em: 18 jun. 2019.

BRASIL. Decreto n. 9.203, de 22 de novembro de 2017. Dispõe sobre a política de governança da administração pública federal direta, autárquica e fundacional. Lex:

Presidência da República. Casa Civil. Subchefia para assuntos jurídicos. Brasília, 22 nov. 2017.

CMMAD Comissão Mundial sobre Meio Ambiente e Desenvolvimento. Nosso futuro comum.

Tradução de Our common future. Rio de Janeiro: Editora da Fundação Getúlio Vargas, 1988.

CNUMAD CONFERÊNCIA DAS NAÇÕES UNIDAS SOBRE O MEIO AMBIENTE E

DESENVOLVIMENTO, 1995. Agenda 21. Tradução: ministério das Relações Exteriores.

Biblioteca Digital da Câmara dos Deputados. Brasília, 1995. Disponível em: <

http://bd.camara.gov.br/bd/handle/bdcamara/7706 >. Acesso em: 7 abr. 2019.

COUTO, E. A. Aplicação dos indicadores de desenvolvimento sustentável da norma ABNT NBR ISO 37120:2017 para a cidade do Rio de Janeiro e análise comparativa com cidades da América Latina. Rio de Janeiro: UFRJ, 2018.

FREY, K. Governança urbana e participação pública. RAC-Eletrônica, v. 1, n. 1, art. 9, p. 136150, jan./abr. 2007. Disponível em: <http://www.anpad.org.br/rac-e>. Acesso em: 14 jun. 2019.

FURTADO, J. S. Indicadores de sustentabilidade e governança. Revista Intertox de Toxicologia, Risco Ambiental e Sociedade, São Paulo, vol. 2, n. 1, p.121-188, fev., 2009.

GOMES, M. L.; MARCELINO, M. M.; ESPADA, M. Proposta para um sistema de indicadores de desenvolvimento sustentável. Portugal: Direcção Geral do Ambiente, 2000. Disponível em: <https://www.apambiente.pt/_zdata/Divulgacao/Publicacoes/SIDS/SIDSPortugal_Proposta20 00.pdf>. Acesso em: 19 dez. 2017.

ONU ORGANIZAÇÃO DAS NAÇÕES UNIDAS. Mais de 90\% da população brasileira viverá em cidades em 20130. ONU News, 2016. Disponível em:

<https://news.un.org/pt/story/2016/10/1566241-mais-de-90-da-populacao-brasileira-viveraem-cidades-em-2030>. Acesso em: 03 abr. 2019.

PALUDO, A. V. Administração pública: teorias e questões. 2. ed. Rio de Janeiro: Elsevier, 2012. $519 \mathrm{p}$.

PROGRAMA CIDADES SUSTENTÁVEIS. Plataforma Cidades Sustentáveis. Disponível em: < https://indicadores.cidadessustentaveis.org.br/>. Acesso em: 04 abr. 2019. 